\title{
Etudes des transferts d'assimilats entre tiges de luzerne (Medicago sativa L.) par l'utilisation du ${ }^{14} \mathrm{CO}_{2}$
}

\author{
L. Dufour 1 , M. Faucher 2 et G. Lemaire ${ }^{1}$ \\ 1 INRA, Station d'écophysiologie des plantes fourragères, 86600 Lusignan; \\ 2 CNRS, Laboratoire de physiologie végétale, UA 574, Station de Beau-Site, 86000 Poitiers, France
}

(reçu le 30-7-1987, accepté le 19-1-1989)

Résumé - Des études antérieures ont montré que la repousse d'une plante de luzerne fait intervenir 2 types morphologiques de tiges:

- les tiges 1 apparaissent les premières, à partir des bourgeons axillaires des bases des tiges laissées par la coupe;

- les tiges 2 prennent naissance directement sur le collet et sont plus vigoureuses que les tiges 1.

La question d'un éventuel rôle trophique des tiges 1 dans la croissance des tiges 2 s'est posée. Pour tenter d'y répondre, après une étude morphologique de la croissance de la plante, le cheminement d'assimilats radioactifs à partir d'une tige 1 ayant assimilé du ${ }^{14} \mathrm{CO}_{2}$ est étudié. II s'avère que les exportations à partir de cette tige 1 sont très réduites, en particulier en début de cycle, et se font principalement vers les racines. II y a donc une relative indépendance trophique entre les tiges au cours d'une repousse.

$\mathrm{CO}_{2}$ marqué - compétition - translocation d'assimilats - morphogenèse - luzerne

Summary - Study of assimilate translocations between stems of lucerne plant using ${ }^{14} \mathrm{CO}_{2}$ labelling. During regrowth of alfalfa plant 2 morphological kinds of stems appear :

- the stems 1 first appeared on the basis remainders of stems left after cutting;

- the stems 2 directly originated on the crown; they were more vigourous than stems 1 and provided the greatest part of the fodder's dry matter at the harvest.

The question of a possible trophical role played by the stems 1 in the growth of the stems 2 was quoted. Then, after a morphological study of the plant growth, the translocation of radioactive assimilates from a stem 1 that has assimilated ${ }^{14} \mathrm{CO}_{2}$ was followed. The exportations out of this stem 1 were very reduced especially at the beginning of the regrowth, and were mainly directed towards the roots. So, there is a relative trophical independance between the stems during one regrowth.

labelled $\mathrm{CO}_{2}$ - competition - assimilate translocation - morphogenesis - lucerne

\section{Introduction}

La luzerne (Medicago sativa L.) est une plante fourragère pérenne qui est exploitée par des coupes successives. Après la coupe, l'interception de la lumière par la plante est quasi nulle et, pendant une période plus ou moins longue, l'efficience de I 'interception est inférieure à sa valeur maximale. La vitesse de croissance de la plante est directement liée à la vitesse d'expansion de l'indice foliaire (Gosse et al., 1984) ainsi qu'à l'utilisation des réserves racinaires (Demarly, 1957). D'un point de vue morphologique, après une coupe, la repousse est assurée par la croissance de 2 types de bourgeons (Fig. 1) (Leach, 1968 et 1970 ; Gosse et al., 1988) :

- des bourgeons préexistants, situés sur la base des tiges laissées après la coupe; nous les appellerons «bourgeons 1 »;

- des bourgeons néo-formés, ou bourgeons blancs, situés au niveau du collet; nous les appellerons «bourgeons 2".

En peuplement dense (c'est-à-dire dans les conditions normales de culture), des bourgeons 1 entrent en croissance tout de suite après la cou- 


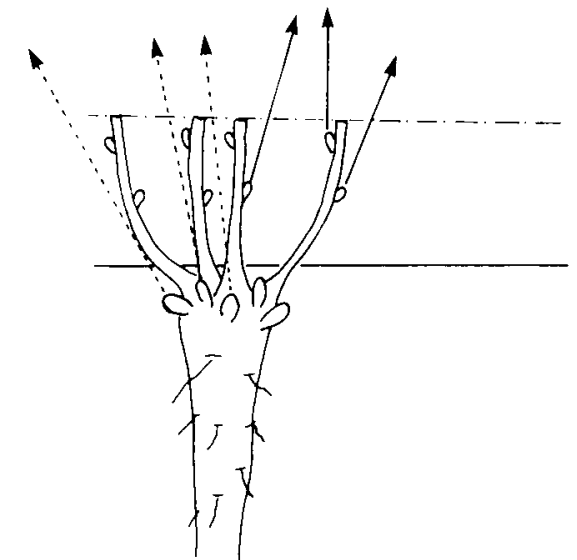

Fig. 1. Schéma des deux types de bourgeons et de tiges présents chez la luzerne. Les tiges $1(-)$ poussent à partir des bourgeons des bases des tiges après la coupe et les tiges 2 (--) à partir du collet.

pe, du fait de la levée de la dominance apicale; les bourgeons 2 ne se développent qu'après quelques jours de latence. Cependant, au bout d'une quinzaine de jours de repousse, les tiges issues de bourgeons 2 sont plus nombreuses et plus vigoureuses que les tiges issues de bourgeons 1; parmi ces dernières, certaines disparaissent, les autres restent assez courtes. Ceci explique que les tiges 2 représentent $80 \%$ de la matière sèche à la récolte pour la variété "Europe" (Gosse et al., 1988).

Suite à cette constatation, on peut se demander si les tiges 1 jouent un rôle trophique en début de repousse pour assurer la croissance ultérieure des tiges 2 . Pour tenter d'élucider ce problème, nous avons choisi de suivre le cheminement d'assimilats radioactifs, depuis la partie distale d'une tige issue de bourgeon 1 ayant assimilé du ${ }^{14} \mathrm{CO}_{2}$, vers les autres organes de la plante. Ainsi, notre objectif était de mettre en évidence un éventuel transfert direct entre la tige 1 marquée (ayant assimilé le ${ }^{14} \mathrm{CO}_{2}$ ) et les tiges 2 en croissance ou, au contraire, une relative indépendance trophique des tiges.

Chez les légumineuses fourragères pérennes comme la luzerne (Caldwell et al., 1984; Cralle et Heichel, 1985), les trèfles blancs et violets (Ryle et al., 1981) les feuilles hautes exportent aussi vers le collet, les racines et les nodosités. En effet, la demande en assimilats est importante car la présence des réserves carbonées dans le pivot est nécessaire pour assurer la pérennité de la plante. De plus, les pertes par respiration sont élevées au niveau des racines et des nodosités, aussi bien chez les légumineuses annuelles (Minchin et Pate, 1973 sur Pisum sativum), que chez les légumineuses pérennes (Witty et al., 1983 sur Medicago sativa). Le marquage de la partie haute d'une tige permet donc de suivre d'éventuels tranferts vers d'autres parties de la plante.

\section{Matériel et méthodes}

\section{Matériel végétal et conditions de culture}

Les plantes de la variété “Europe" sont cultivées en serre dans des pots de $7 \mathrm{~cm}$ de diamètre et $40 \mathrm{~cm}$ de hauteur, sur du sable fin. On distingue 4 lots différents par la date de semis et le nombre de coupes subies (Tableau 1). Sur les 4 lots, la première coupe a été effectuée au stade "pleine floraison». L'arrosage est effectué avec une solution nutritive sans azote: $0,14 / 1$ $\mathrm{K}_{2} \mathrm{HPO}_{4} ; 0,20 \mathrm{~g} / \mathrm{l} \mathrm{MgSo}{ }_{4}, 7 \mathrm{H} 20 ; 0,22 \mathrm{~g} / \mathrm{l} \mathrm{CaC1} 1_{2}$; $0,07 \mathrm{~g} / / \mathrm{K}_{2} \mathrm{SO}_{4}$; oligoéléments: $\mathrm{FeSO}_{4} 4,96 \mathrm{mg} / \mathrm{l} ; \mathrm{Na}_{2}$ EDTA $6,45 \mathrm{mg} / \mathrm{l} ; \mathrm{CuSO}_{4}, 5 \mathrm{H}_{2} \mathrm{O}, 56 \mathrm{mg} / \mathrm{l} ; \mathrm{ALSO}_{4} 56$ $\mathrm{mg} / \mathrm{l} ; \mathrm{LiCl} 28 \mathrm{mg} / \mathrm{l}, \mathrm{ZnSO} \mathrm{C}_{4}, 56 \mathrm{mg} / \mathrm{l} ; \mathrm{H}_{3} \mathrm{BO}_{3} 0,611 \mathrm{mg} /$; $\mathrm{SnCL}_{2}, \mathrm{H}_{2} \mathrm{O}, 28 \mathrm{mg} / \mathrm{l}$.

En début d'expérience, les plantes sont coupées à $7 \mathrm{~cm}$ de la surface du sable sauf 11 d'entre elles issues des 4 lots qui sont coupées à $0 \mathrm{~cm}$. Toutes les plantes sont placées en chambre de culture dans les conditions suivantes : $25^{\circ} \mathrm{C}$ pendant les $14 \mathrm{~h}$ d'éclairement et $20^{\circ} \mathrm{C}$ la nuit; hygrométrie de $65 \%$. L'éclairement utile à la photosynthèse, dispensé par des lampes à vapeur de mercure (Osram 400W) est d'environ $130 \mu \mathrm{mol} \mathrm{m} \mathrm{m}^{-2} \mathrm{~s}^{-1}$. Dans la salle de culture la densité est relativement faible $\left(100\right.$ plantes $\left.\mathrm{m}^{-2}\right)$ alors qu'au champ elle atteint 200 plantes $\mathrm{m}^{-2}$.

\section{Etude morphologique de la repousse}

Deux fois par semaine, des mesures non destructives sont réalisées sur chaque plante. Elles concernent le nombre de tiges 1 et de tiges 2 et la longueur des tiges; les tiges 1 sont mesurées de leur point d'insertion sur les tiges résiduelles à l'apex, les tiges 2 de la surface du sable à l'apex. La distinction entre tiges 1 et tiges 2 n'est pas toujours aisée; aussi, la notation réalisée à l'arrachage est plus fiable.

On relève également le poids de matière sèche à la dernière coupe (tiges 1 et 2) et à l'arrachage de la plante (racines, tiges 1 et 2 ).

\section{Expériences d'assimilation du ${ }^{14} \mathrm{CO}_{2}$}

Le dispositif expérimental est celui décrit par Faucher et Bonnemain (1981). Six plantes sont utilisées simultanément. La partie d'une tige 1 (environ $15 \mathrm{~cm}$ ) de chaque plante est placée dans la chambre d'assimilation où circule, pendant $3 \mathrm{~h}$, le ${ }^{14} \mathrm{CO}_{2}$ dégagé par la réaction de $1 \mathrm{ml}$ de $\mathrm{H}_{2} \mathrm{SO}_{4} 10 \%$ sur $25 \mu \mathrm{Ci}$ de $\mathrm{Na}_{2}{ }^{14}$ $\mathrm{CO}_{3}$ soit $925 \mathrm{kBq}$ (CEA-Service des molécules marquées; activité spécifique: $203,5.10^{4} \mathrm{kBq} / \mathrm{mMol}$ ). A la fin des fixations de ${ }^{14} \mathrm{CO}_{2}$, le circuit est modifié afin que l'excédent de ${ }^{14} \mathrm{CO}_{2}$ soit piégé par de la soude.

Les fixations de ${ }^{14} \mathrm{CO}_{2}$ débutent toujours en fin de matinée pour éviter les éventuelles fluctuations journalières de photosynthèse et du transport des assimilats. Les plantes étaient soumises à l'éclairement naturel sous serre. Puis, pour chaque série, une plante est arrachée afin de déterminer la répartition des substances ${ }^{-14} \mathrm{C}$ après $3 \mathrm{~h}$ de chasse; les 5 autres plantes sont remises en chambre de culture pour une période variable (Tableau II). Un traitement “ombrage" (Tableau II) visant à simuler la concurrence pour la lumière en couvert végétal dense est réalisé en plaçant un grillage plastique, qui arrête $50 \%$ de la lumière, au-dessus des plantes.

A l'arrachage, on sépare chaque plante en différentes parties: tiges 1 marquées, tiges 1 non mar- 
Tableau I. Caractéristiques des différents lots de luzerne utilisés pour les expériences.

\begin{tabular}{lllll}
\hline Lots & $\begin{array}{l}\text { Nombre } \\
\text { de } \\
\text { plantes }\end{array}$ & $\begin{array}{l}\text { Date } \\
\text { de } \\
\text { semis }\end{array}$ & $\begin{array}{l}\text { Nombre de coupes } \\
\text { subies avant } \\
\text { l'assimilation de }{ }^{14} \mathrm{CO}_{2}\end{array}$ & $\begin{array}{l}\text { Age des plantes } \\
\text { a la dernière coupe } \\
(26 / 05 / 86) \text { en jours }\end{array}$ \\
\hline 1 & 8 & $17 / 01 / 86$ & 4 & 160 \\
2 & 16 & $30 / 01 / 86$ & 3 & 147 \\
3 & 23 & $19 / 02 / 86$ & 3 & 127 \\
4 & 24 & $01 / 03 / 86$ & 2 & 118 \\
\hline
\end{tabular}

quées, tiges 2 et racines. Chaque partie est congelée rapidement entre deux plaques métalliques prérefroidies placées dans un congélateur $\left(-26^{\circ} \mathrm{C}\right)$ puis lyophilisée (lyophilisateur Edwards, modèle EF2), avant d'être pesée et broyée. Six fractions aliquotes de $\mathbf{5 0}$ mg sont prélevées pour chaque partie et placées chacune dans un flacon à scintillation liquide en vue de déterminer la quantité de radioactivité. La solubilisation des molécules radioactives est assurée par $100 \mu \mathrm{l}$ d'acide perchlorique à $70 \%$ auxquels on ajoute $200 \mu$ d'eau oxygénée à $30 \%$ qui décolore la solution (Delrot et Geiger, 1986). Après passage à l'étuve à $60^{\circ}$ $\mathrm{C}$ pendant une nuit, on ajoute $5 \mathrm{ml}$ de liquide scintillant (PCS II-Amersham). Le comptage en scintillation liquide (compteur intertechnique Abac SL 33) est fait avec correction de quenching par la méthode de l'étalonnage externe.

\section{Résultats et discussion}

\section{Analyse morphologique}

Deux problèmes ont rendu cette étude délicate : les effectifs réduits et l'hétérogénéité entre plantes, l'arrachage échelonné à partir du $15^{\circ}$ jour après la coupe des plantes ayant assimilé le ${ }_{14} \mathrm{CO}_{2}$. Ces plantes n'ont pas pu être prises au hasard pour des raisons de montage expérimental. En effet, nous avons choisi des plantes possédant des tiges 1 suffisamment longues pour pouvoir être introduites dans la chambre d'assi- milation sans difficultés. Or il se trouve que ces plantes possédaient également un nombre de tiges 1 supérieur à la moyenne.

\section{1) Evolution du nombre de tiges par plante}

Dans nos conditions de culture (100 plantes $\mathrm{m}^{2}$, donc faible compétition pour la lumière), le nombre de tiges 1 reste longtemps supérieur au nombre de tiges 2 (Fig. 2) et il n'y a pas de disparition de tiges 1 ; la légère diminution observée à partir du $15^{\mathrm{e}}$ jour de repousse (Fig. 2) n'est attribuée qu'à des problèmes d'échantillonnage (cf. remarques ci-dessus). Ces résultats sont différents de ceux obtenus au champ (peuplement dense donc plus forte compétition pour la lumière) où, au fur et à mesure du développement des tiges 2 , le nombre de tiges 1 diminue et devient inférieur au nombre de tiges 2 (Gosse et al., 1988).

D'autre part, dans nos conditions expérimentales, les tiges 2 apparaissent tout de suite après la coupe, sans le temps de latence observé au champ (Fig. 2). Pour les plantes coupées à $0 \mathrm{~cm}$ du sol (sans bourgeons 1), les tiges 2 apparaissent plus vite et sont plus nombreuses que les plantes coupées à $7 \mathrm{~cm}$ (avec bourgeons 1) (Fig. 2). II semble donc que le développement des bourgeons 1 inhibe en partie celui des bourgeons 2.

Tableau II. Calendrier des assimilations et des récoltes.

\begin{tabular}{lllllllll}
$N^{\circ}$ Assimilation & 1 & 2 & 3 & 4 & 5 & 6 & 7 & 8 \\
\hline $\begin{array}{c}\text { Assimilation } \\
\text { (nombre de jours à } \\
\text { partir de la coupe) }\end{array}$ & -3 & 14 & 15 & 16 & 17 & 29 & 30 & 31 \\
$\begin{array}{c}\text { Temps de transport } \\
\text { jours) }\end{array}$ & 17 & 13 & 1 & 1 & 13 & 1 & 6 & 13 \\
$\begin{array}{c}\text { Récolte (nombre de } \\
\text { jours après la coupe }\end{array}$ & 14 & 27 & 16 & 17 & 30 & 30 & 36 & 44 \\
Traitement & Lumière & Lumière & Ombrage & Lumière & Ombrage & Lumière & Lumière Lumière \\
\hline
\end{tabular}




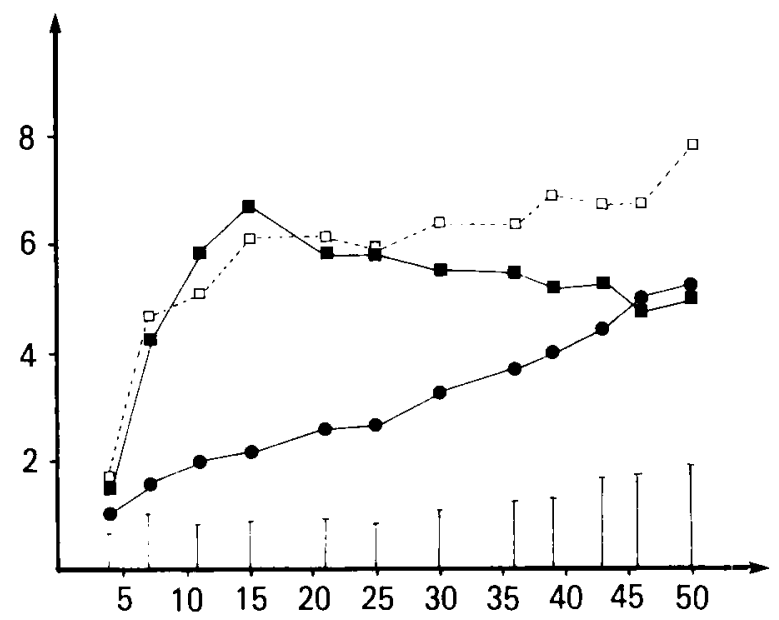

Fig. 2. Evolution du nombre moyen de tiges 1 et 2 par plante. Coupe à $7 \mathrm{~cm}$ : tiges 1; tiges 2; coupe à $0 \mathrm{~cm}$ : $\square$ tiges 2 . Les barres verticales représentent la ppds $(P<0,05)$. Abscisse : nombre de jours après la coupe; ordonnée : nombre de tiges par plante.

\section{2) Croissance en longueur des tiges}

Après une coupe à $7 \mathrm{~cm}$, les bourgeons 2 entrent en croissance tout au long de la repousse et les tiges 2 sont toujours plus longues, en moyenne, que les tiges 1 (Fig. 3). Mais, en couvert végétal dense, à la fin de la repousse précédente, ces bourgeons 2 reçoivent peu de lumière et restent sous forme de bourgeons blancs; ils ne se développent qu'après la coupe mais avec un temps de latence, ce qui explique la longueur moindre des tiges 2 par rapport aux tiges 1 en début de repousse (Gosse et al., 1988).

\section{3) Production de matière sèche}

Les moyennes des poids de matière sèche par plante sont les suivantes, à la coupe du 26 mai 1986 :

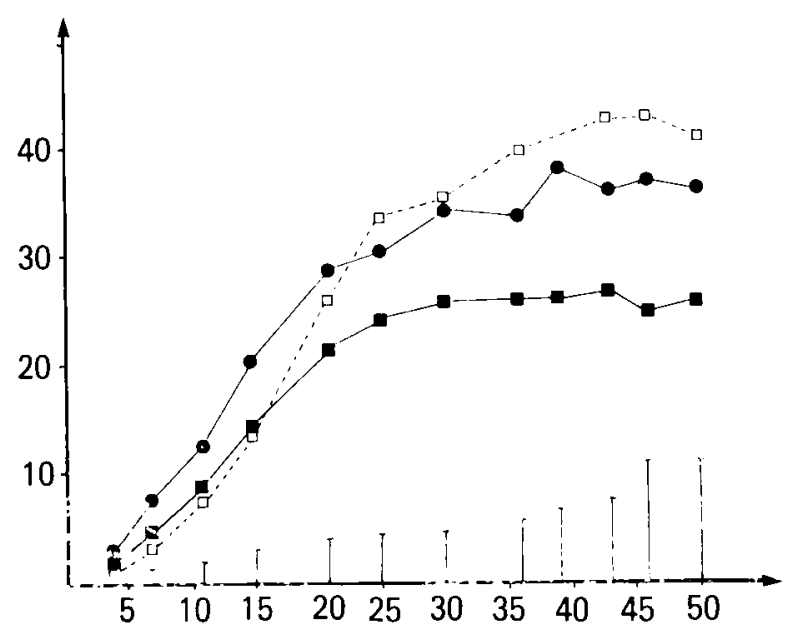

Fig. 3. Evolution de la longueur moyenne des tiges après une coupe. Coupe à $7 \mathrm{~cm}$ : tiges 1 ; tiges 2; coupe à $0 \mathrm{~cm}: \square$ tiges 2. Les barres verticales représentent la ppds $(P<0,05)$. Abscisse : nombre de jours après la coupe; ordonnée : longueur moyenne des tiges $(\mathrm{cm})$.
$-1,97 \pm 0,24 \mathrm{~g}$ pour l'ensemble des tiges 1 ;

$-1,62 \pm 0,28 \mathrm{~g}$ pour l'ensemble des tiges 2 .

En plantes isolées, les tiges 2 produisent à peu près la moitié de la matière sèche aérienne de la plante, alors qu'en condition de compétition, elles en produisent $80 \%$ (Gosse et al., 1988). L'absence de mortalité parmi les tiges 1 dans nos conditions peut expliquer en partie cette différence.

\section{Mesures de radioactivité}

\section{1) Marquage au ${ }^{14} \mathrm{C}$ avant la coupe}

Un lot de 6 plantes assimile le ${ }^{14} \mathrm{CO}_{2} 3$ jours avant la dernière coupe (Tableau II) et les mesures de radioactivité sont réalisées 14 jours après cette coupe. La radioactivité restante se répartit entre les racines, les tiges $t$ et les tiges 2 (Tableau III). II y a autant de radioactivité dans les tiges 1 qui se sont développées sur le reliquat de la tige 1 marquée que dans la totalité des autres tiges 1 (Tabl. 3). Une certaine quantité d'assimilats marqués resterait dans la partie basse de la tige au moment de la coupe et irait directement aux bourgeons 1 qui se développent sur cette tige lors de la repousse.

\section{2) Marquage au ${ }^{14} \mathrm{C}$ après la coupe}

La quantité de radioactivité exportée par la tige marquée est faible (20\% maximum); elle augmente avec le temps de transport (Tableaux IV et V). L'ombrage réduit légèrement les transferts d'assimilats marqués dans les différentes parties de la plante (Tableau IV), ce qui est en accord avec les résultats de Ryle et Powell (1976) sur graminées. L'ombrage n'augmente pas la proportion de ${ }^{14} \mathrm{C}$ utilisé par les tiges 2.

L'exportation est plus forte pour les plantes marquées en fin de repousse (Tableau $V$ ) que pour celles marquées en milieu de repousse (Tableau IV). Dans tous les cas, les racines sont les organes qui reçoivent le plus de radioactivité. En fin de repousse, il y a une mise en réserve d'assimilats dans les racines et dans la base des tiges. Le pourcentage de radioactivité retrouvé dans les tiges 1 et 2 est faible. Cette radioactivité pourrait provenir d'assimilats utilisés dans les racines pour l'élaboration des acides aminés à partir de l'azote fixé au niveau des nodosités.

\section{Discussion}

D'après nos résultats, les tiges 1 ne paraissent pas participer directement à la croissance des tiges 2. Au contraire, elles semblent même inhiber le démarrage de certains bourgeons 2 puisqu'une coupe à $0 \mathrm{~cm}$ augmente le nombre de tiges 2 (Fig. 2). 
Tableau III. Mesures de radioactivité pour les plantes marquées 3 jours avant la coupe (moyenne de 6 plantes, 17 jours de transport). NB : «tiges 1 " représente l'ensemble des tiges 1 sauf celles issues de la tige marquée. $\mathrm{RSA}=\frac{\text { (Radioactivité/poids de matière sèche) (organe) }}{\text { (Radioactivité/poids de matière sèche) (plante) }}$

\begin{tabular}{lccc}
\hline Organes & $\begin{array}{l}\text { \% de la radioactivité } \\
\text { retrouvée }\end{array}$ & $R S A$ & $\begin{array}{c}\text { Radioactivité totale (plante à la } \\
\text { récolte moyenne de 6 plantes) }\end{array}$ \\
\hline $\begin{array}{l}\text { Tiges 1 issues de } \\
\text { la tige marquée }\end{array}$ & 27,19 & 8,19 & $3,7 \mu \mathrm{Ci}$ \\
$\begin{array}{l}\text { Tiges 1 } \\
\text { Tiges 2 }\end{array}$ & 29,82 & 1,25 & soit \\
Racines & 8,77 & $133,2 \mathrm{kBq}$ \\
\hline
\end{tabular}

Tableau IV. Pourcentage de radioactivité pour les plantes marquées en milieu de repousse. Les chiffres entre parenthèses sont les valeurs absolues exprimées en $\mathrm{kBq}$.

\begin{tabular}{|c|c|c|c|c|c|}
\hline \multirow[b]{2}{*}{ Organes } & \multicolumn{4}{|c|}{ Temps de transport / traitement } & \multirow[b]{2}{*}{$\begin{array}{l}13 \text { jours } \\
\text { Ombrage }\end{array}$} \\
\hline & $\begin{array}{l}3 \text { heures } \\
\text { Lumière }\end{array}$ & $\begin{array}{l}1 \text { jour } \\
\text { Lumière }\end{array}$ & $\begin{array}{l}1 \text { jour } \\
\text { Ombrage }\end{array}$ & $\begin{array}{l}11 \text { jours } \\
\text { Lumière }\end{array}$ & \\
\hline Tige 1 marquée & $\begin{array}{l}99,76 \\
(2,522)\end{array}$ & 99,04 & 99,66 & 93,31 & 96,93 \\
\hline Autres tiges 1 & $\begin{array}{l}0,02 \\
(0,0005)\end{array}$ & 0,54 & 0,07 & 2,17 & 0,73 \\
\hline Tiges 2 & $\begin{array}{l}0,02 \\
(0,0004)\end{array}$ & 0,03 & 0,02 & 1,16 & 0,69 \\
\hline Racines & $\begin{array}{l}0,21 \\
(0,005)\end{array}$ & 0,41 & 0,26 & 3,83 & 1,79 \\
\hline
\end{tabular}

Tableau V. Pourcentage de radioactivité pour les plantes marquées en fin de repousse. Les chiffres entre parenthèses sont les valeurs absolues exprimées en $\mathrm{kBq}$.

\section{Temps de transport / traitement}

\begin{tabular}{|c|c|c|c|c|}
\hline Organes & $\begin{array}{l}3 \text { heures } \\
\text { Lumière }\end{array}$ & $\begin{array}{l}1 \text { jour } \\
\text { Lumière }\end{array}$ & $\begin{array}{l}6 \text { jours } \\
\text { Lumière }\end{array}$ & $\begin{array}{l}13 \text { jours } \\
\text { Lumière }\end{array}$ \\
\hline Tige 1 marquée & $\begin{array}{l}99,68 \\
(1,607)\end{array}$ & 90,01 & 87,32 & 79,87 \\
\hline Autres tiges 1 & $\begin{array}{l}0,08 \\
(0,0005)\end{array}$ & 0,94 & 1,23 & 5,15 \\
\hline Tiges 2 & $\begin{array}{l}0,02 \\
(0,0004)\end{array}$ & 0,27 & 3,39 & 2,32 \\
\hline Racines & $\begin{array}{l}0,22 \\
(0,003)\end{array}$ & 8,78 & 8,07 & 12,66 \\
\hline
\end{tabular}


Dans nos conditions expérimentales, c'est-àdire en l'absence de compétition pour la lumière, toutes les tiges ont accès à la lumière. Chacune d'entre elles fixe le carbone dont elle a besoin et peut également participer à la constitution des réserves racinaires contribuant ainsi au démarrage des bourgeons après la coupe.

En revanche, d'après les résultats de Gosse et al., (1988), en couvert végétal dense, du fait de la compétition pour la lumière, seules les tiges les plus hautes (tiges 2) ont accès à la lumière. Les tiges 1, plus courtes, disparaissent car elles ne reçoivent pas assez de lumière pour assimiler tout le carbone nécessaire à leur croissance.

C'est seulement en fin de croissance que l'exportation de ${ }^{14} \mathrm{CO}_{2}$ à partir des tiges 1 devient notable dans nos conditions. L'étude d'un éventuel transfert lors de la sénescence des tiges 1 que l'on observe dans un couvert dense au champ (Gosse et al., 1988) mériterait d'être réalisée afin de savoir quelle est son importance dans la mise en réserve racinaire.

Enfin la question du déterminisme de la vigueur supérieure des tiges 2 reste posée; estelle sous la dépendance de phénomènes hormonaux ou résulte-t-elle d'une plus grande facilité d'accès aux réserves du pivot consécutive à la position plus basale bourgeons 2 par rapport aux bourgeons 1 ?

\section{Conclusion}

La repousse des plantes de luzerne de la variété «Europe» est différente sur le plan morphologique selon que la culture est réalisée en couvert dense ou en plante isolée, c'est-à-dire en présence ou en absence de compétition pour la lumière. En plante isolée, les tiges 1 sont présentes pendant toute la repousse alors qu'au champ elles disparaissent en grand nombre. Cependant, elles ne semblent pas participer directement au développement des bourgeons 2 puisque l'exportation des assimilats ${ }^{14} \mathrm{C}$ à partir d'une tige 1 est réduite et se fait essentiellement vers les racines. Ce résultat doit permettre une analyse plus détaillée des mécanismes de compétition pour la lumière entre tiges et entre plantes dans un peuplement dense de luzerne. En effet, s'il y a indépendance trophique entre les tiges, on peut alors admettre que la position d'une tige dans le couvert et donc son éclairement sont les seuls facteurs à prendre en compte pour estimer son bilan de carbone et ses chances de survie au cours de la repousse. Les plantes les plus résistantes à la compétition devraient alors être celles qui émettent davantage de tiges 2 que de tiges 1 . Une analyse de la variabilité génétique pour ce caractère devrait être envisagée.

\section{Références}

Caldwell C.D., Fensom D.S., Bordeleau L., Thompson R.G., Drouin R. \& Didsbury R. (1984) Translocation of $13 \mathrm{~N}$ and ${ }^{11 \mathrm{C}}$ between nodulated roots and leaves in alfalfa seedlings. J. Exp. Bot. 35, 431-443

Cralle H.T. \& Heichel G.H. (1985) Interorgan photosynthate partitioning in alfalfa. Plant Physiol. 79, 3, 381 385

Delrot S. \& Geiger D.R. (1986) Comparison of binding and release of $N$-ethylmaleimide and $p$-chloromercuribenzenesulphonic acid by leaf tissues. Physiol. Veg. 24, 355-365

Demarly Y. (1957) Biologie et exploitation de la luzerne. Ann. Amelior. Plant. 7, 3, 247-272

Faucher M. \& Bonnemain J.L. (1981) Action des barrières thermiques sur la circulation libérienne chez le maïs (Zea mays L.). Physiol. Veg. 19, 379-390

Gosse G. , Chartier M. \& Lemaire G. (1984) Mise au point d'un modèle de prévision de production pour une culture de luzerne. C.R. Acad. Sci. Paris 289, Série III, $18,541-544$

Gosse G, Lemaire G., Chartier M. \& Balfourier F. (1988) Structure of a lucerne population (Medicago sativa L.) and dynamics of stem competition for light during regrowth. J. Appl. Ecol. 25, 609-617

Leach G.J. (1968) The growth of lucerne plant cutting at different stages of maturity and at different intensities. Aust. J. Agric. Res., 19, 4, 517-530

Leach G.J. (1970) Shoot growth of lucerne plants cut at different heights. Aus. J. Agric. Res., 21, 4, 583-591

Minchin F.R. \& Pate J.S. (1973) The carbon balance of a legume and the functional economy of its root nodules. J. Exp. Bot., 24, 79, 259-271

Ryle G.J.A. \& Powell C.E. (1976) Effect of rate of photosynthesis on the pattern of assimilate distribution in the graminaceous plant. J. Exp. Bot., 27, 97, 189-199

Ryle G.J.A., Powell C.E. \& Gordon A.J. (1981) Patterns of ${ }^{14} \mathrm{C}$ labelled assimilate partioning in red and white clover during vegetative growth. Ann. Bot. 47, 4 505-514

Witty J., Minchin F.R. \& Sheehy J.E. (1983) Carbon costs of nitrogenase activity in legume roots nodules determined using acetylen and oxygen. J. Exp. Bot. $34,951-963$ 